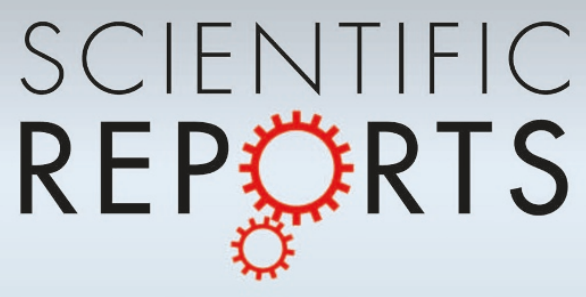

OPEN

SUBJECT AREAS:

NUCLEAR ASTROPHYSICS

SUPERHEAVY ELEMENTS

THEORETICAL NUCLEAR PHYSICS

EXPERIMENTAL NUCLEAR

PHYSICS

Received

27 June 2013

Accepted

5 September 2013

Published

24 September 2013

Correspondence and requests for materials should be addressed to

S.F.S. (shuifa.shen@ fds.org.cn)

\section{High-Spin States and Level Structure in Stable Nucleus Strontium-84}

\author{
Shuifa Shen 1,2,3,4, Guangbing Han ${ }^{5}$, Shuxian Wen ${ }^{6}$, Xuzhong Kang ${ }^{7}$, Yupeng Yan ${ }^{2,4}$, Zhijun Bai ${ }^{3}$, \\ Yican $\mathrm{Wu}^{1}$, Xiaoguang $\mathrm{Wu}^{6}$, Lihua Zhu ${ }^{8}$, Guangsheng $\mathrm{Li}^{6} \&$ Chuangye $\mathrm{He}^{6}$
}

'Institute of Nuclear Energy Safety Technology, Chinese Academy of Sciences, Hefei 23003 1, China, ${ }^{2}$ School of Physics, Institute of Science, Suranaree University of Technology, Nakhon Ratchasima 30000, Thailand, ${ }^{3}$ State Key Laboratory of Nuclear Physics and Technology (Peking University), Beijing 100871, China, ${ }^{4}$ Thailand Center of Excellence in Physics (ThEP), Commission on Higher Education, Thailand, ${ }^{5}$ School of Physics, Shandong University, Jinan 250100, China, ${ }^{6}$ China Institute of Atomic Energy, P. O. Box 275(10), Beijing 102413, China, ${ }^{7}$ Shanghai Institute of Applied Physics, Chinese Academy of Sciences, Shanghai 201800, China, ${ }^{8}$ School of Physics and Nuclear Energy Engineering, Beihang University, Beijing 100191, China.

High-spin states of ${ }^{84} \mathrm{Sr}$ are populated through the reaction ${ }^{70} \mathrm{Zn}\left({ }^{18} \mathrm{O}, 4 \mathrm{n}\right){ }^{84} \mathrm{Sr}$ at the beam energy of $75 \mathrm{MeV}$. The measurements of excitation functions, $\gamma-\gamma$ coincidences, directional correlations of oriented states (DCO) ratios and $\gamma$-transition intensities are performed using eight anticompton HPGe detectors and one planar HPGe detector. Based on the experimental results, we establish a new level scheme of ${ }^{84} \mathrm{Sr}$, in which 12 new states and nearly 30 new $\gamma$-transitions are identified in the present work. The positive-parity yrast band is extended to spin $I^{n}=24^{+}$, while one negative-parity band is extended to spin $I^{\pi}=19^{-}$and it is found that the even-spin and odd-spin members in high-spin states show the nature of signature staggering. The deformation of ${ }^{84} \mathrm{Sr}$ is studied by calculating the total-Routhian-surfaces (TRS) of positive-parity yrast states in the cranked shell model formalism.

N uclei with $\mathrm{Z} \approx 40$ and $\mathrm{N} \approx 45$ lie in a transitional region between the deformed nuclei and spherical ones. There exist collective bands in the nuclei such as some isotopes of $\mathrm{Sr}, \mathrm{Zr}$ and $\mathrm{Mo}$, and their structures also have single-particle features. The neutron-deficient strontium isotopes such as ${ }^{76,78} \mathrm{Sr}$ are predicted to be deformed in the ground state with $\beta_{2}=0.421^{1}$. On the other hand, ${ }^{88} \mathrm{Sr}^{2}$ with a closed neutron shell at $\mathrm{N}=50$ and a fairly good proton-subshell closure at $Z=38$, is found to be doubly magic and, hence, spherical. ${ }^{84} \mathrm{Sr}$, which has four neutron holes away from ${ }^{88} \mathrm{Sr}$, should display both collective and quasiparticle excitations ${ }^{3}$; this makes it an interesting object of investigation. Dewald et al. ${ }^{3}$, Kucharska et al. ${ }^{4}$, Lister et al. ${ }^{5}$, and Chattopadhyay et al. ${ }^{6}$ have investigated the structure of ${ }^{84} \mathrm{Sr}$ successively. More than ten years ago, low-spin states of ${ }^{80,84} \mathrm{Sr}$ have been studied by Döring et al. ${ }^{7}$ whose decay data suggested that the low-lying structures of ${ }^{80,84} \mathrm{Sr}$ show many vibration-like features in a potential with modest deformation including candidates for two- and three-phonon multiplets. This vibration-like nature seems to evolve to a more rotational behavior with increasing angular momentum $I$ and decreasing neutron number $N$. So in order to study the structure feature of ${ }^{84} \mathrm{Sr}$ in detail, the present experiment is designed to extend the level scheme of ${ }^{84} \mathrm{Sr}$ to higher spin states. To analyze the deformation for the positive-parity state of this nucleus, total Routhian surface (TRS) calculations are carried out by means of the pairing-deformation-frequency self-consistent cranked shell model. Finally, the properties of the negative-parity bands of ${ }^{84} \mathrm{Sr}$ are also discussed qualitatively.

\section{Results}

All events are sorted off-line into a $4096 \times 4096$ disc stored matrix on which the energy gates are set later. According to already existing level scheme ${ }^{6}$, we gate first on the strongest $793 \mathrm{keV} \gamma$-ray, and find that all of the relevant $\gamma$-transitions given in Ref. 6 are observed. Gated then on each of these relevant $\gamma$-transitions, the cascade relationship revealed in Ref. 6 is confirmed. In the coincidence spectrum gated by the $793 \mathrm{keV} \gamma$-transition, we also observe relatively strong 706, 759, and $988 \mathrm{keV} \gamma$-transitions, etc., and find that these $\gamma$-transitions are clearly visible in the coincidence spectrum gated by the $1001 \mathrm{keV} \gamma$-transition, as shown in Fig. 1(a). In order to confirm these $\gamma$-transitions belonging to the negative-parity band of ${ }^{84} \mathrm{Sr}$, we gate on the $988 \mathrm{keV} \gamma$-transition, and clearly observe $510,793,974$, and $1001 \mathrm{keV} \gamma$-transitions, etc., which have already been confirmed to belong to ${ }^{84} \mathrm{Sr}$, but fail to observe other confirmed $\gamma$-transitions of the positive-parity band. In addition, we also observe relatively strong 759 and $1041 \mathrm{keV} \gamma$-transitions, etc., in the coincidence spectrum gated by the $988 \mathrm{keV} \gamma$-ray. In the 

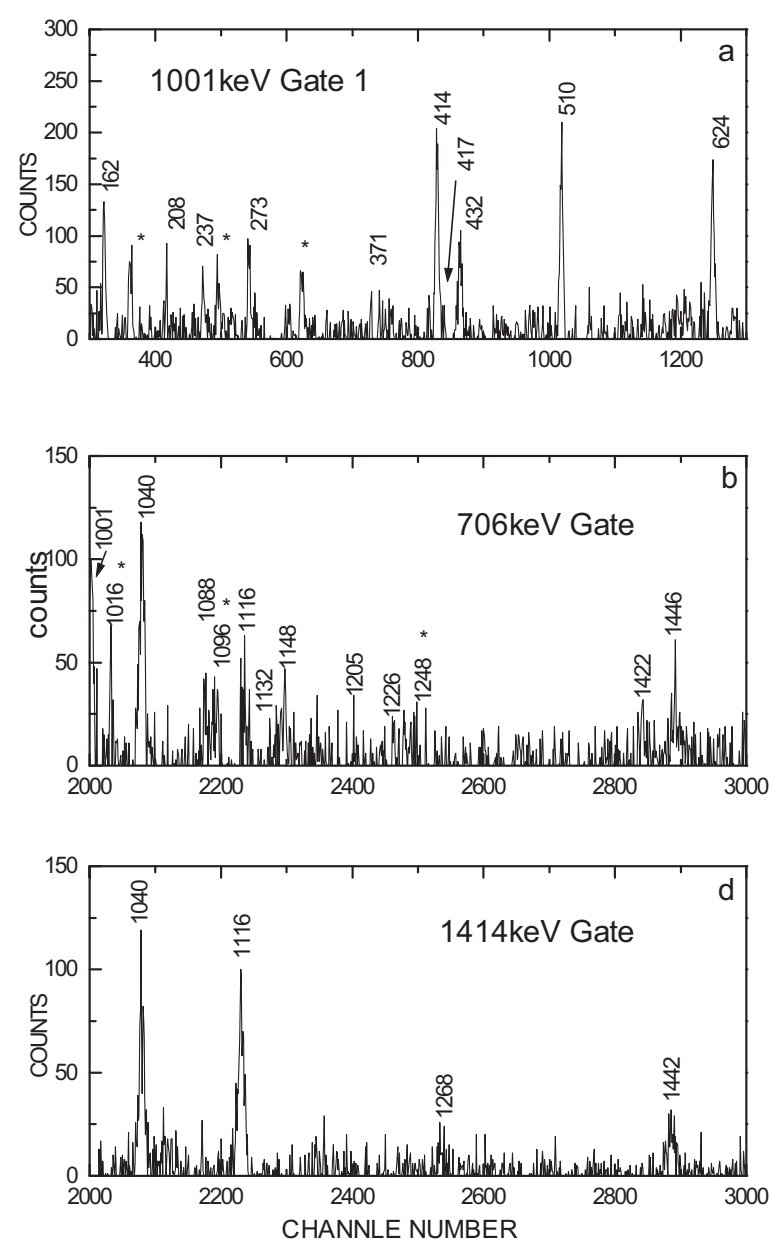
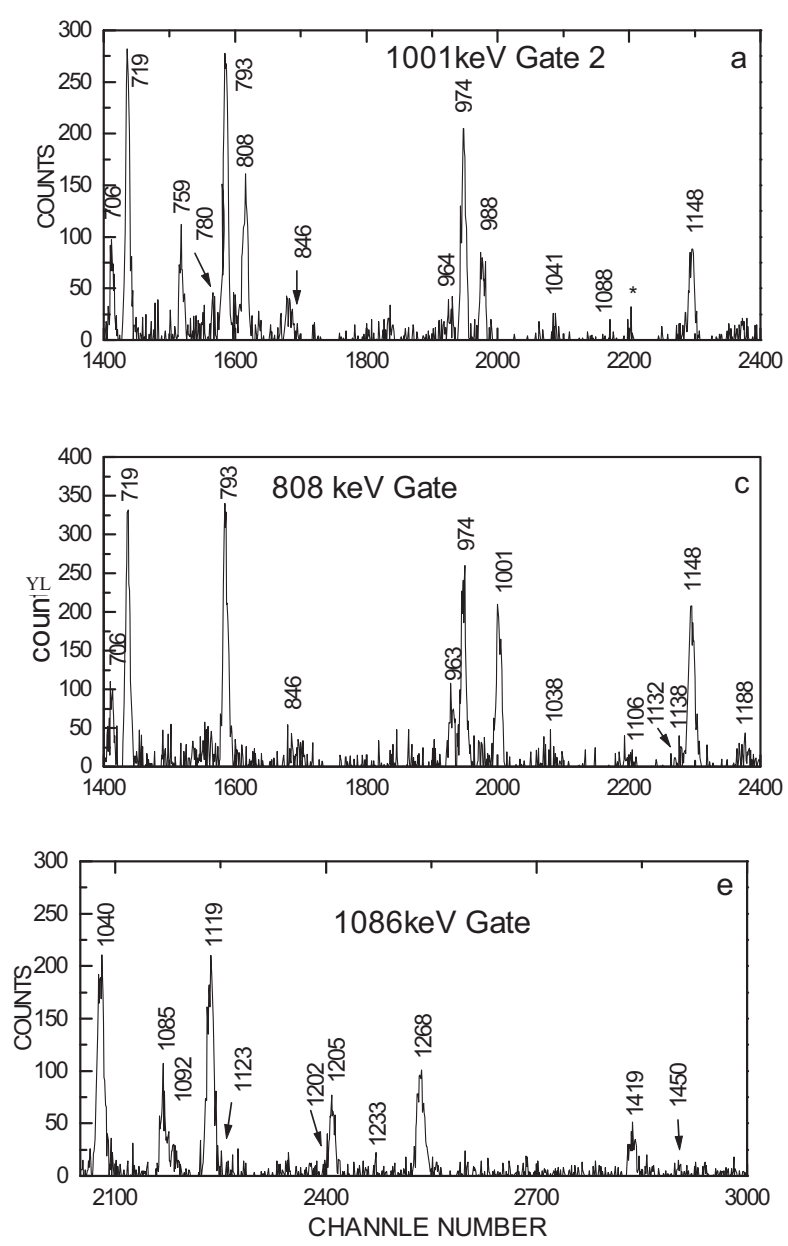

Figure 1 Some examples of coincidence spectra gated by (a) 1001, (b) 706, (c) 808, (d) 1414, and (e) $1086 \mathrm{keV} \gamma$-rays, respectively. The asterisk denotes the ray that does not belong to ${ }^{84} \mathrm{Sr}$.

coincidence spectrum gated by $510 \mathrm{keV}$, we observe relatively strong 759, 988, and $1041 \mathrm{keV} \gamma$-transitions, etc., besides the 208 and $417 \mathrm{keV} \gamma$-transitions. It can be seen that the 759, 988, and $1041 \mathrm{keV} \gamma$-transitions belong to ${ }^{84} \mathrm{Sr}$ and their cascade relationship with the $510 \mathrm{keV} \gamma$-transition of the negative-parity band is confirmed. In the coincidence spectra gated by both the 759 and $719 \mathrm{keV} \gamma$-transitions, we observe the relatively strong $780 \mathrm{keV} \gamma$ transition in each case. From the fact that the sum of 719 and 780 equals the sum of 510 and 988 , the cascade order of the 988 and $759 \mathrm{keV} \gamma$-transitions can be obtained. At the same time, we observe 624 and $417 \mathrm{keV} \gamma$-transitions in the coincidence spectrum gated by the $759 \mathrm{keV} \gamma$-transition, so their cascade order is also assigned. In the coincidence spectrum gated by the $719 \mathrm{keV} \gamma$-transition, we observe the strong $162 \mathrm{keV} \gamma$-transition. Gated on the $162 \mathrm{keV} \gamma$ transition, we prove that it has a cascade relationship with the 706, 719, and $1088 \mathrm{keV} \gamma$-transitions.

So far, the cascade relationships of strong $\gamma$-transitions observed in the coincidence spectrum gated by $793 \mathrm{keV}$ are almost all assigned. In particular, the $706 \mathrm{keV} \gamma$-transition should be mentioned here. We can observe the strong $706 \mathrm{keV} \gamma$-transition in the coincidence spectra gated by $524 \mathrm{keV} \gamma$-ray of the positive-parity band and $1001 \mathrm{keV} \gamma$-ray, respectively. The $706 \mathrm{keV} \gamma$-ray can also be observed in the coincidence spectrum gated by the $624 \mathrm{keV} \gamma$-ray, and its intensity is much stronger than that of 162 and $1088 \mathrm{keV} \gamma$ rays, etc., in this spectrum. These indicate that the $706 \mathrm{keV} \gamma$-ray exists in two different cascades. The placements of $706 \mathrm{keV} \gamma$-transitions are confirmed through analyzing the coincidence spectrum gated by itself, shown in Fig. 1(b). In addition, we observe the strong
$964 \mathrm{keV} \gamma$-transition and a string of cascade transitions that are established above it in the coincidence spectrum gated by the $808 \mathrm{keV} \gamma$-transition, as shown in Fig. 1(c).

In the coincidence spectrum gated by the $524 \mathrm{keV} \gamma$-transition of the positive-parity band, we observe not only 1202, 1205, and $1418 \mathrm{keV} \gamma$-transitions, but also 1414 and $1442 \mathrm{keV} \gamma$-transitions, and moreover find that the intensity of the $1414 \mathrm{keV} \gamma$-transition is stronger than that of the $1442 \mathrm{keV} \gamma$-transition. Note that this intensity relationship also appears in the coincidence spectra gated by the 1040 and $1116 \mathrm{keV} \gamma$-transitions, one may conclude that there exists a cascade relationship between the $1414 \mathrm{keV}$ and $1442 \mathrm{keV} \gamma$ transition. Fig. 1(d) shows the high-energy cascade part of the coincidence spectrum gated by $1414 \mathrm{keV}$. In the coincidence spectrum gated by $1086 \mathrm{keV}$, we observe not only 325, 1085, 1094, and $1274 \mathrm{keV} \gamma$-transitions which Lister et al. ${ }^{5}$ have already presented, but also 1450, 1752, and $2017 \mathrm{keV} \gamma$-rays, shown in Fig. 1(e). In the coincidence spectra gated by the 1268 and $1418 \mathrm{keV} \gamma$-transitions, we also observe 1450 and $1752 \mathrm{keV} \gamma$-transitions, and thus confirm their cascade order. In summary, 12 new levels and nearly 30 new $\gamma$ transitions are identified in the present work. Due to the weak intensities of the 1752 and $2017 \mathrm{keV} \gamma$-transitions, we use dashed lines to denote them in the level scheme proposed in the present work.

As mentioned above, in order to assign the multipolarity of these $\gamma$-transitions and then the spins and parities of the relevant states, the detectors are positioned around $38^{\circ}$ (at $35^{\circ}, 36^{\circ}, 39^{\circ}, 43^{\circ}$, and $143^{\circ}$ with respect to the beam direction) and around $90^{\circ}$ (at $81^{\circ}, 90^{\circ}$, and $102^{\circ}$ with respect to the beam direction) for deducing the DCO ratios. We in general gate on the strong E2 $\gamma$-transitions when the 
Table I | Some new $\gamma$-transitions of ${ }^{84} \mathrm{Sr}$, the corresponding initial level energies, initial state spins, final state spins, and their DCO ratios deduced from the present experiment

\begin{tabular}{|c|c|c|c|c|c|c|c|c|c|}
\hline$E_{x}(\mathrm{keV})$ & $E_{\gamma}(\mathrm{keV})$ & $I_{i}$ & $I_{f}$ & DCO & $E_{x}(\mathrm{keV})$ & $E_{\gamma}(\mathrm{keV})$ & $I_{i}$ & $I_{f}$ & DCO \\
\hline 3278 & 510.1 & $6^{-}$ & $5^{-}$ & $0.46^{\mathrm{al}}$ & 7619 & 1138.1 & $15^{-}$ & $13^{-}$ & $0.33^{e)}$ \\
\hline 3487 & 208.4 & $7^{-}$ & $6^{-}$ & $0.66^{\mathrm{b}}$ & 7823 & 1084.5 & $15^{+}$ & $14^{+}$ & $0.69^{\mathrm{dl}}$ \\
\hline 3649 & 162.2 & $7^{-}$ & $7^{-}$ & $0.88^{c}$ & 8006 & 182.4 & $16^{+}$ & $15^{+}$ & 0.64 \\
\hline 4266 & 780.1 & $8^{-}$ & $7^{-}$ & & 8139 & 1226.3 & $16^{-}$ & $14^{-}$ & $0.10^{\mathrm{e}}$ \\
\hline 4266 & 988.8 & $8^{-}$ & $6^{-}$ & $0.85^{\mathrm{al}}$ & 8752 & 1132.1 & $\left(16^{-}\right)$ & $15^{-}$ & $1.08^{\mathrm{e}}$ \\
\hline 4533 & 1202.0 & $10^{+}$ & $8^{+}$ & $0.91^{\mathrm{d}}$ & 8752 & 1240.0 & $\left(16^{-}\right)$ & $14^{-}$ & $0.37^{\mathrm{e}}$ \\
\hline 4737 & 1088.1 & $9^{-}$ & $7^{-}$ & $0.75^{\circ}$ & 9065 & 1446.2 & $17^{-}$ & $15^{-}$ & $0.56^{\dagger}$ \\
\hline 5025 & 759.1 & $10^{-}$ & $8^{-}$ & $0.95^{\circ}$ & 9097 & 1093.6 & $17^{+}$ & $16^{+}$ & $0.34^{d \mid}$ \\
\hline 5443 & 417.1 & $11^{-}$ & $10^{-}$ & & 9097 & $1274.4^{[5]}$ & $17^{+}$ & $15^{+}$ & $0.76^{\mathrm{d})}$ \\
\hline 5861 & 1414.0 & $12^{+}$ & $10^{+}$ & & 9424 & 325.4 & $18^{+}$ & $17^{+}$ & \\
\hline 6067 & 1041.1 & $12^{-}$ & $10^{-}$ & $1.07^{c}$ & 10487 & 1422.1 & $\left(19^{-}\right)$ & $17^{-}$ & $0.69^{f}$ \\
\hline 6407 & 964.0 & $12^{-}$ & $11^{-}$ & $0.78^{\mathrm{b}}$ & 10547 & 1123.0 & $19^{+}$ & $18^{+}$ & $0.48^{\mathrm{d}}$ \\
\hline 6481 & 74.2 & $13^{-}$ & $12^{-}$ & & 10547 & 1449.7 & $19^{+}$ & $17^{+}$ & $1.00^{\mathrm{d})}$ \\
\hline 6913 & 846.3 & $14^{-}$ & $12^{-}$ & $0.43^{e l}$ & 11062 & 514.1 & $20^{+}$ & $19^{+}$ & \\
\hline 7303 & 1442.0 & $14^{+}$ & $12^{+}$ & & 12299 & 1238.1 & $\left(21^{+}\right)$ & $20^{+}$ & $0.58^{\mathrm{dl}}$ \\
\hline 7512 & 1030.1 & $14^{-}$ & $13^{-}$ & $0.94^{e l}$ & 12299 & 1752.0 & $(21+)$ & $19^{+}$ & $1.00^{d)}$ \\
\hline 7512 & 1105.0 & $14^{-}$ & $12^{-}$ & $0.82^{b)}$ & 12920 & 1858.4 & $22^{+}$ & $20^{+}$ & $1.05^{\mathrm{d}, \mathrm{g})}$ \\
\hline 7619 & 108.2 & $15^{-}$ & $14^{-}$ & & 14937 & 2017.1 & $\left(24^{+}\right)$ & $22^{+}$ & $1.06^{d, g l}$ \\
\hline 7619 & 706.0 & $15^{-}$ & $14^{-}$ & $0.92^{\text {e) }}$ & & & & & \\
\hline
\end{tabular}

a), b), c), d), e), and f) gated by $793,808,719,1086,625$, and $706 \mathrm{keV}$, respectively, when DCO ratios are deduced; g) obtained from the spectrum without subtracting the background.

DCO ratios are extracted. In order to get better statistics, we also gate on M1 (E1) $\gamma$-transitions (e.g. 625 and $706 \mathrm{keV}$ ) in the analysis. The DCO ratios deduced from the present work are basically consistent with the multipolarity derived by Chattopadhyay et al. ${ }^{6}$. We list in Table I only the DCO ratios of the new $\gamma$-transitions measured in the present experiment and the spins and parities of the corresponding initial and final states. Listed in Table II are the relative intensities of a part of the $\gamma$-transitions, obtained by using the computer code SPAN98 (The relative intensities of the $\gamma$-rays are normalized to the intensity of the $793 \mathrm{keV}$ transition, which is set to $\mathrm{I}_{\gamma}=100$ ). In order to reduce the interference of nearby $\gamma$-rays, i.e., to improve accuracy, we obtain the intensity of the $974 \mathrm{keV} \gamma$-transition relative to the $793 \mathrm{keV} \gamma$-transition in the total coincidence projected spectrum at first, then obtain the intensities of other $\gamma$-transitions relative to the $974 \mathrm{keV} \gamma$-transition in the coincidence spectrum gated by the $793 \mathrm{keV} \gamma$-transition, and at last normalize the relative intensities of all these $\gamma$-transitions to the intensity of the $793 \mathrm{keV}$ transition, which is set to $I_{\gamma}=100$. The $\gamma$-transitions whose relative intensities are less than $2 \%$ are not listed in Table II. Based on the results above, a partial level scheme of ${ }^{84} \mathrm{Sr}$ is given in Fig. 2, including the results of the present and previous works. It can be noted that the order in the level scheme, for the 414, 432 and $706 \mathrm{keV} \gamma$ rays, shown in Fig. 2 is not consistent with that of the work carried out by García Bermúdez et al. ${ }^{9}$, but among them the order of 414 and $432 \mathrm{keV} \gamma$ rays is consistent with that reported by Chattopadhyay et al. ${ }^{6,10}$.

\section{Discussion}

The level scheme is compared with its neighboring even-even isotopes ${ }^{78,80,82,86} \mathrm{Sr}$. We find that $\mathrm{N}=45$ and $\mathrm{N}=46$ divide the structures of the nuclei with $\mathrm{Z}=38$ into two kinds of different properties, i.e., the structures of the nuclei with $\mathrm{N}<45$ exhibit rotational behavior, whereas the nuclei with $\mathrm{N}>46$ show strong vibrational behavior, see Refs. 11-13 for details.

The positive-parity states are extended to spin $24^{+}$, especially four odd-spin states $15^{+}, 17^{+}, 19^{+}$, and $\left(21^{+}\right)$are detected in the present work. As shown in Fig. 2, the ground-state band of ${ }^{84} \mathrm{Sr}$ displays a certain degree of collectivity, just as its neighboring isotones. In the present work the observation of $\gamma$-transitions between the close-lying similar spin states of these two positive-parity bands demonstrates the existence of neutron-proton interaction, which is in agreement with the results of Chattopadhyay et al. ${ }^{6}$. All these are in good agreement with the calculation results carried out by Dewald et al. based upon interacting boson model (IBM) ${ }^{3}$ and those of Chattopadhyay et al. ${ }^{6}$. When the spins and parities of ${ }^{84} \mathrm{Sr}$ reach above $14^{+}$, it displays the properties of the rotational structure of deformed nuclei. The signature staggering for the $\gamma$-transition energies within this band above $14^{+}$can be observed. It can be seen that it no longer maintains the shape of the ground state, i.e., the shape change occurs and then the rotation happens. It is worthy to mention that on the top of $14^{+}$ ${ }^{84} \mathrm{Sr}$ still exhibits the dominance of E2 $\gamma$-transitions, whereas ${ }^{88} \mathrm{Mo}$ and ${ }^{86} \mathrm{Zr}$ transfer to the strong M1 $\gamma$-transitions ${ }^{6}$.

\begin{tabular}{|c|c|c|c|c|c|c|c|c|c|}
\hline$E_{\gamma}(\mathrm{keV})$ & 86 & 272 & 348 & 372 & 414 & 432 & 510 & 524 & 609 \\
\hline $\mathrm{I}_{\gamma}$ & $9.8(6)$ & $2.0(4)$ & $8.3(6)$ & $1.5(4)$ & $14.3(6)$ & $6.5(6)$ & $10.9(7)$ & $40.8(8)$ & $3.0(2)$ \\
\hline $\mathrm{E}_{\mathrm{x}}(\mathrm{keV})$ & 4533 & 3040 & 3679 & 3649 & 6481 & 6913 & 3278 & 3331 & 3649 \\
\hline $\mathrm{l}_{\gamma}$ & $10.6(6)$ & $6.2(6)$ & $9.5(6)$ & $12.5(6)$ & $2.5(4)$ & 100 & $10.1(6)$ & $24.3(7)$ & $18.6(7)$ \\
\hline $\mathrm{E}_{\mathrm{x}}(\mathrm{keV})$ & 6067 & 3487 & 7619,5443 & 3487 & 5025 & 793 & 5443 & 4533 & 3679 \\
\hline $\mathrm{E}_{\gamma}(\mathrm{keV})$ & 964 & 974 & 988 & 997 & 1001 & 1040 & 1085 & 1088 & 1116 \\
\hline $\mathrm{E}_{\gamma}(\mathrm{keV})$ & 1119 & 1148 & 1205 & 1268 & 1442 & 1446 & & & \\
\hline $\mathrm{l}_{\gamma}$ & $14.1(5)$ & $5.5(4)$ & $2.1(4)$ & $0.8(2)$ & $0.8(2)$ & $1.4(2)$ & & & \\
\hline $\mathrm{E}_{x}(\mathrm{keV})$ & 5652 & 4635 & 5652 & 8006 & 7303 & 9065 & & & \\
\hline
\end{tabular}




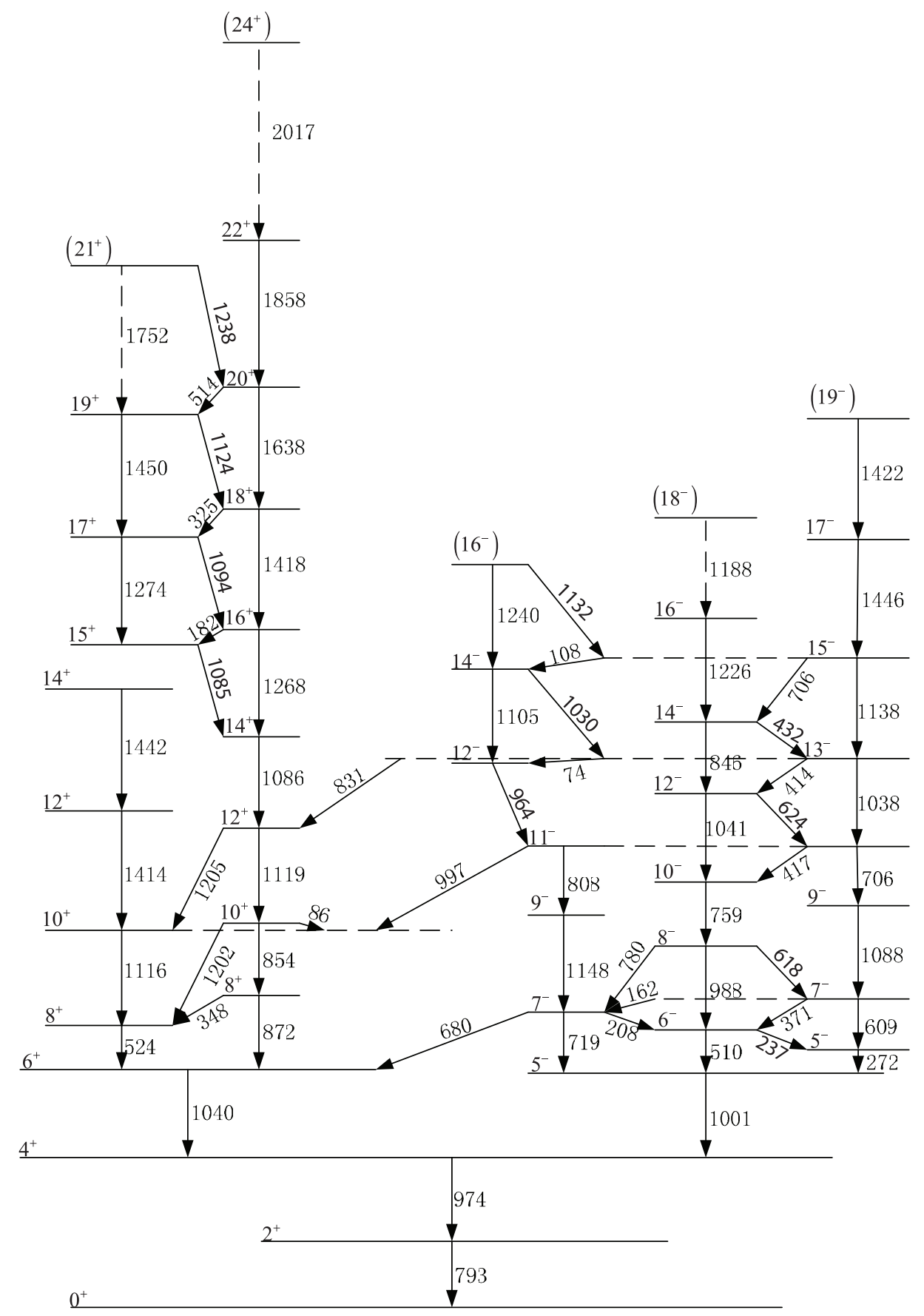

Figure $2 \mid$ The partial level scheme of ${ }^{84} \mathrm{Sr}$ proposed in the present work. The transition energies are given in units of keV.

To analyze the deformation for the positive-parity states of this nucleus in detail, total-Routhian-surface (TRS) calculations are carried out by means of the pairing-deformation-frequency self-consistent cranked shell model ${ }^{14,15}$, i.e., pairing and deformation changing as a function of rotational frequency are determined self-consistently. In practical calculations, for a given frequency and deformation, pairing is treated self-consistently by solving the cranked pairingcorrelated Hamiltonian and then the shape of a given state is determined by minimizing the calculated TRS (for details, see Refs. 14, 15). The single-particle energies are obtained with the nonaxial deformed Woods-Saxon (WS) potential ${ }^{16}$. Particle number projection is approximated by the Lipkin-Nogami (LN) approach ${ }^{14,17,18}$. Both monopole and quadrupole pairings ${ }^{19-21}$ are considered with the monopole pairing strength $G$ determined by the average-gap method $^{22}$ and quadrupole strengths obtained by restoring the Galilean invariance of the paired many-body Hamiltonian ${ }^{19,20}$. The quadrupole pairing has a negligible effect on energies but has an important influence on the collective rotation ${ }^{15,23}$. In the cranked model calculation ${ }^{14,15}$, the nuclear energy at zero frequency is calculated by the Strutinsky method ${ }^{24}$ with the standard liquid-drop energy $^{25}$. Calculations are performed in the lattice of quadrupole $\left(\beta_{2}, \gamma\right)$ deformations with the hexadecapole $\beta_{4}$ variation. Samples of TRS are presented in Fig. 3 in the polar coordinate plane $\left(\beta_{2}, \gamma\right)$ at specific rotational frequencies $\hbar \omega=0.2,0.4,0.6$, and $0.8 \mathrm{MeV}$ corresponding to a spin range of $I \sim(0-20) \hbar$, and the energy difference between neighboring contours is $200 \mathrm{keV}$. According to our TRS calculations, at a low rotational frequency, this nucleus is predicted to have an almost spherical shape and be soft in the $\beta_{2}$ and $\gamma$ 


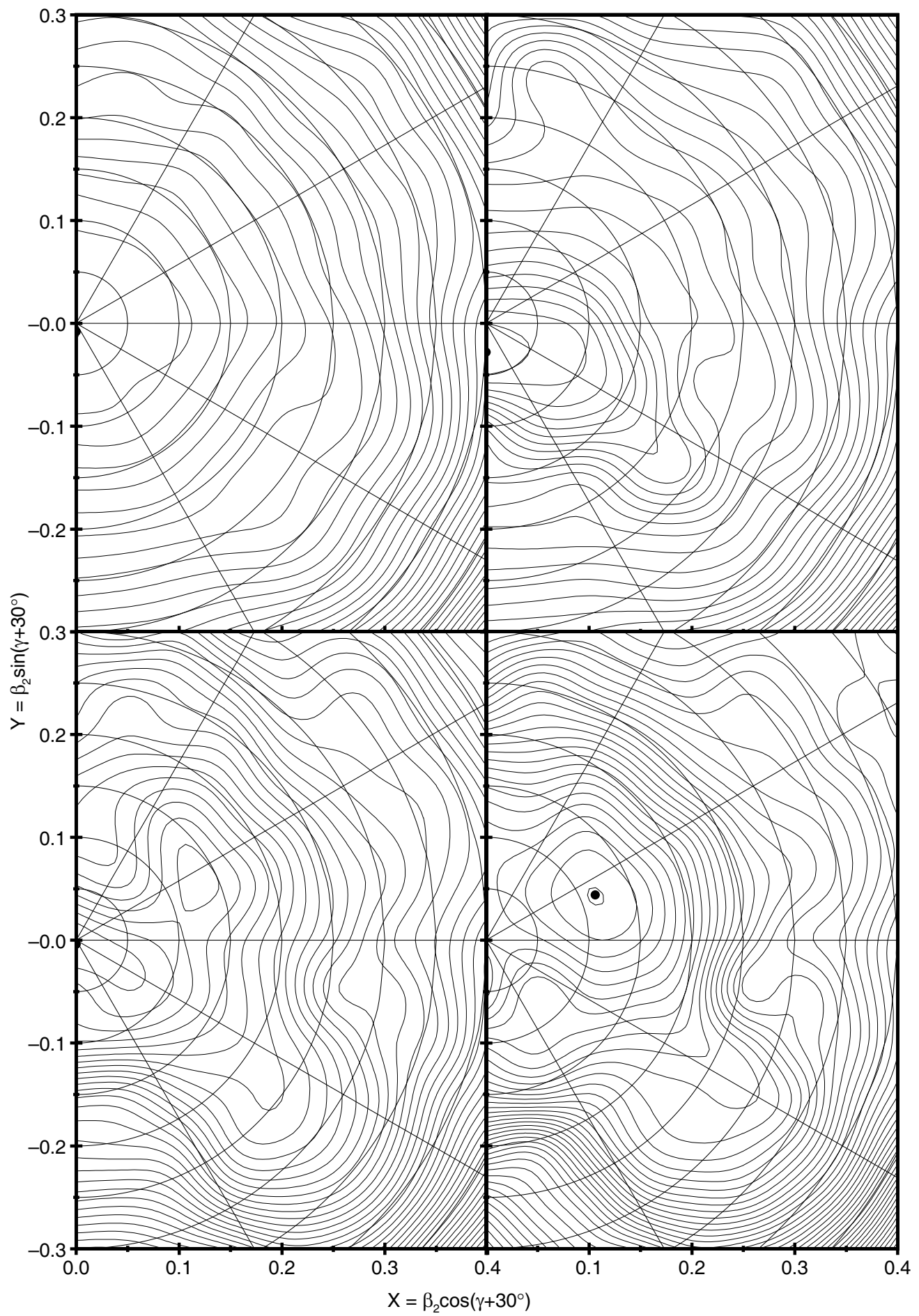

Figure $3 \mid$ Total-Routhian-surfaces for ${ }^{84} \mathrm{Sr}$ positive-parity states at $\hbar \omega=0.2$ (upper left), 0.4 (upper right), 0.6 (lower left), and $0.8 \mathrm{MeV}$ (lower right) corresponding to $I \sim(0-20) \hbar$. The black dot represents the overall minimum in each panel, and the contour lines are separated by $200 \mathrm{keV}$.

directions. With increasing frequency, the nucleus becomes slightly more rigid and has a deformed shape. In TRS calculations, at rotational frequency $\hbar \omega=0.6 \mathrm{MeV}$, the second minimum appears (see the lower left panel of Fig. 3). This minimum at $\beta_{2}=0.122$ and $\gamma=$ $-4.759^{\circ}$ becomes yrast at $\hbar \omega=0.7 \mathrm{MeV}$ and persists up to very high rotational frequency (see for example the lower right panel of Fig. 3).

The negative-parity states are extended to $10487 \mathrm{keV}$. The first negative-parity band consisting of $5^{-}, 7^{-}, 9^{-}$, and $11^{-}$levels is not extended very high. We find only one $964 \mathrm{keV} \mathrm{M1} \gamma$-transition feeding the $11^{-}$state in this band. We take the 414,432 , and
$624 \mathrm{keV} \mathrm{M1} \gamma$-transitions, which Chattopadhyay et al. ${ }^{6}$ have already assigned through the lifetime measurements, as a part of a band based on the second $5^{-}$level. The $6^{-}, 7^{-}$, and $8^{-}$states which lie above the second $5^{-}$level have already been discovered by Dewald et $\mathrm{al}^{3}{ }^{3}$ and Chattopadhyay et $\mathrm{al}^{6}{ }^{6}$, and assigned spins and parities. We extend the negative-parity states to spin of $19^{-}$and energy of $10487 \mathrm{keV}$. It shows that the $5^{-}, 6^{-}, 7^{-}$, and $8^{-}$states indeed exhibit rotational behavior. This is consistent with the prediction of Dewald et al. ${ }^{3}$. But the M1 $\gamma$-transitions between the $10^{-}, 9^{-}$, and $8^{-}$states are not obvious; this phenomenon is consistent with the results by means 


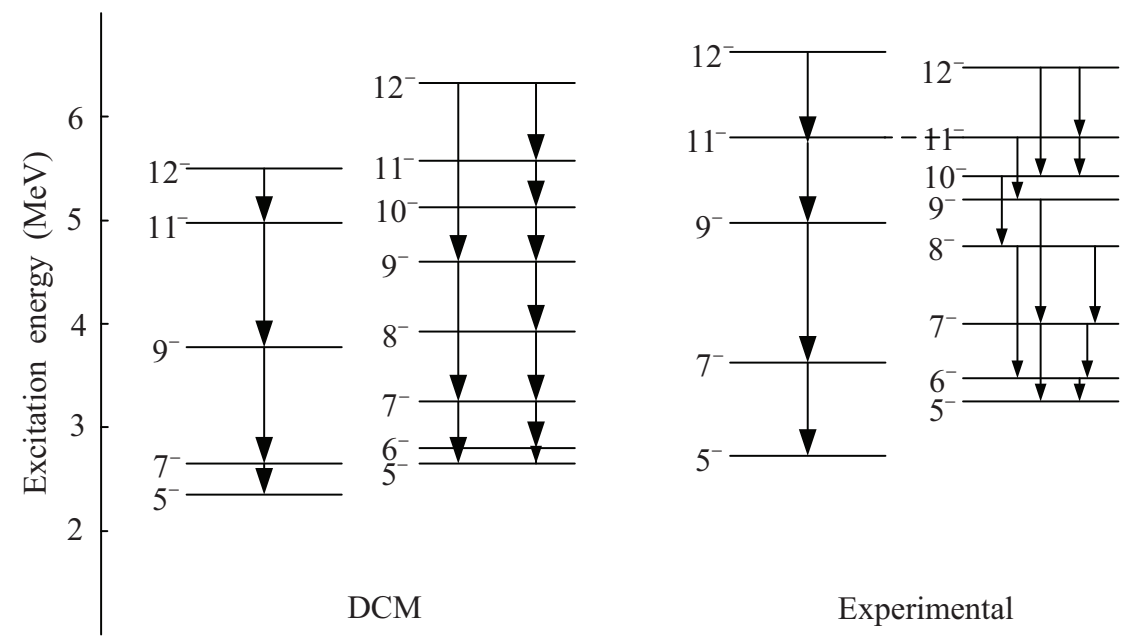

Figure $4 \mid$ DCM calculations for negative-parity states in ${ }^{84} \mathrm{Sr}$ compared with the present experimental data.

of the deformed configuration-mixing shell model (DCM) calculations in the work of $\mathrm{Sahu}^{26}$, who suspect the prediction made by Dewald et al. ${ }^{3}$. The states above $11^{-}$exhibit a very good collective vibration-like nature while the even-spin and odd-spin members of this band show the nature of signature staggering for the states above $14^{-}$.

As shown in Fig. 4, the levels of the negative-parity bands proposed in the present work are in agreement with DCM calculations, but the calculations haven't given higher spin states. It should be noted that the $\mathrm{B}(\mathrm{M} 1) / \mathrm{B}(\mathrm{E} 2)$ ratios show an abrupt increase above the $11^{-}$state, e.g. $\mathrm{B}(780) / \mathrm{B}(988)=0.38\left(\mu_{\mathrm{N}} / \mathrm{eb}\right)^{2}$, compared with $\mathrm{B}(624) / \mathrm{B}(1041)=28.24\left(\mu_{\mathrm{N}} / \mathrm{eb}\right)^{2}$. The observed increase in the $\mathrm{B}(\mathrm{M} 1) / \mathrm{B}(\mathrm{E} 2)$ values in the negative-parity band may probably be attributed to neutron holes occupying the $g_{9 / 2}$ high- $\Omega$ orbitals ${ }^{6}$.

\section{Methods}

The high-spin states of ${ }^{84} \mathrm{Sr}$ are populated through the reaction ${ }^{70} \mathrm{Zn}\left({ }^{18} \mathrm{O}, 4 \mathrm{n}\right){ }^{84} \mathrm{Sr}$ at the projectile energy of $75 \mathrm{MeV}$. The ${ }^{18} \mathrm{O}$ beam is provided by HI-13 Tandem Accelerator at China Institute of Atomic Energy (CIAE). In order to increase the reaction yields in the experiment, the target consists of a stack of two thin selfsupporting ${ }^{70} \mathrm{Zn}$ foils with a thickness of about $400 \mu \mathrm{g} / \mathrm{cm}^{2}$ each. The ${ }^{70} \mathrm{Zn}$ was isotopically enriched to $99.3 \%$. At first the excitation function is measured using beam energies of $67,70,75,80$, and $87 \mathrm{MeV}$, respectively. Then $\gamma-\gamma-t$ ( $t$ refers to the relative time difference between any two coincident $\gamma$ rays detected) coincidence measurements are performed at optimal beam energy of $75 \mathrm{MeV}$ (with beam intensity of I = 8-9 nA) using an array comprised of eight high-purity germanium (HPGe) detectors each with a BGO anti-Compton shield and one planar HPGe detector; each $\mathrm{BGO}(\mathrm{AC}) \mathrm{HPGe}$ detector has an efficiency of approximately $25 \%$ and the planar HPGe detector $20 \%$. In the present work, in the analysis of the data, spectrum is produced with a gate on the prompt time peak and a time-random background matrix is subtracted. These detectors are placed at angles of $35^{\circ}, 36^{\circ}, 39^{\circ}, 43^{\circ}, 81^{\circ}, 90^{\circ}$, $102^{\circ}, 143^{\circ}$, and $159^{\circ}$ with respect to the beam direction, respectively, so that the directional correlation of oriented states (DCO) ratios can be deduced. The energy resolutions of the Ge detectors are between 1.8 and $2.2 \mathrm{keV}$ at $1.333 \mathrm{MeV} \gamma$-ray energy. A total of $1.8 \times 10^{8}$ double- or higher-fold coincidence events are collected on the magnetic tapes in an event-by-event mode in the present experiment. The trigger condition for data acquisition is set such that events are recorded when at least two $\gamma$ rays in the Ge detectors are in prompt coincidence. The overall count rates collected by this array during the experiment are 500-600 events/s. The $\gamma$-ray energies and relative efficiencies are calibrated with ${ }^{133} \mathrm{Ba}$ and ${ }^{152} \mathrm{Eu}$ sources. In order to determine the multipolarity of the $\gamma$-ray transitions, three detectors near $90^{\circ}$ (at $81^{\circ}, 90^{\circ}$ and $102^{\circ}$ ) with respect to the beam axis are sorted against the other five detectors near $38^{\circ}$ (at $35^{\circ}, 36^{\circ}, 39^{\circ}, 43^{\circ}$ and $143^{\circ}$ ) to produce a two-dimensional angular correlation matrix from which it is possible to extract the average directional correlation of oriented state (DCO) intensity ratios. The $\gamma-\gamma$ coincidence data are analyzed with the Radware software package ${ }^{8}$. The interpretation of DCO ratios is most straightforward when gating is carried out on stretched E2 transitions. In this case DCO ratios of about 1.0 and 0.5 are expected for stretched $\Delta \mathrm{I}=2$ and $\Delta \mathrm{I}=1$ transitions, respectively. Consequently, the inverse value of 2.0 is expected for a quadrupole transition gated on a dipole transition, and it is certainly 1.0 for a dipole transition gated on a dipole transition. If dipole-quadrupole mixing is included, then the DCO ratio for a $\Delta \mathrm{I}=1$ transition may vary between 0.2 and 1.8 depending on the amount of mixing and the nuclear alignment. Ambiguities may also occur since an unstretched pure $\Delta \mathrm{I}=0$ transition is expected to have a ratio slightly larger than 1 , i.e., similar to a stretched $\mathrm{E} 2$ transition.

1. Möller, P., Nix, J. R., Myers, W. D. \& Swiatecki, W. J. Nuclear Ground-State Masses and Deformations. At. Data Nucl. Data Tables. 59, 185-381 (1995).

2. Kumbartzki, G. J. et al. Structure of the $\mathrm{Sr}-\mathrm{Zr}$ isotopes near and at the magic $\mathrm{N}=$ 50 shell from g-factor and lifetime measurements in ${ }_{40}^{80} \mathrm{Zr}$ and ${ }^{84,86,88}{ }_{38} \mathrm{Sr}$. Phys. Rev. C 85, 044322 (2012).

3. Dewald, A. et al. High Spin States of ${ }^{84}$ Sr. Phys. Rev. C 25, 226-239 (1982).

4. Kucharska, A. I., Billowes, J. \& Lister, C. J. g Factors Near the First Backbend in ${ }^{82} \mathrm{Sr}$ and ${ }^{84}$ Sr. J. Phys. G 15, 1039-1051 (1989).

5. Lister, C. J., Chowdhury, P. \& Vretenar, D. The Extension of the I. B. M. to High Spin. Nucl. Phys. A 557, 361c-380c (1993).

6. Chattopadhyay, S. et al. Structure of the Four Quasiparticle Band in ${ }^{84}$ Sr. Phys. Rev. C 50, 93-98 (1994).

7. Döring, J., Aprahamian, A. \& Wiescher, M. Low-Spin States from Decay Studies in the Mass 80 Region. J. Res. Natl. Inst. Stand. Technol. 105, 43-51 (2000).

8. Radford, D. C. ESCL8R and LEVIT8R: Software for interactive graphical analysis of HPGe coincidence data sets. Nucl. Instrum. Methods Phys. Res. A 361, 297-305 (1995).

9. García Bermúdez, G. et al. High Spin States of ${ }^{84}$ Sr. Phys. Rev. C 49, 3309-3311 (1994).

10. Chattopadhyay, S. \& Jain, H. C. Comment on "High Spin States of ${ }^{84}$ Sr". Phys. Rev. C 51, 3532-3533 (1995)

11. Rudolph, D. et al. Systematics of Even-Even $\mathrm{T}(\mathrm{z})=1$ Nuclei in the $\mathrm{A}=80$ Region: High-spin rotational bands in ${ }^{74} \mathrm{Kr},{ }^{78} \mathrm{Sr}$, and ${ }^{82} \mathrm{Zr}$. Phys. Rev. C 56, 98-117 (1997).

12. Müller, H. W. Nuclear Data Sheets for A $=82$. Nucl. Data. Sheets. 50, 17-19 (1987).

13. Fields, C. A. et al. A Study of the ${ }^{84} \operatorname{Kr}(\alpha, 2 \mathrm{n} \gamma)^{86} \mathrm{Sr}$ Reaction. Nucl. Phys. A 398, 512-524 (1983)

14. Satuła, W., Wyss, R. \& Magierski, P. The Lipkin-Nogami Formalism for the Cranked Mean Field. Nucl. Phys. A 578, 45-61 (1994).

15. Satuła, W. \& Wyss, R. Extended Mean Field Description of Deformed States in Neutron Deficient Cd- and Sn-Nuclei. Phys. Scr. T 56, 159-166 (1995).

16. Ćwiok, S., Dudek, J., Nazarewicz, W., Skalski, J. \& Werner, T. R. Single-particle energies, wave functions, quadrupole moments and g-factors in an axially deformed woods-saxon potential with applications to the two-centre-type nuclear problems. Comp. Phys. Comm. 46, 379-399 (1987).

17. Pradhan, H. C., Nogami, Y. \& Law, J. Study of Approximations in the Nuclear Pairing-Force Problem. Nucl. Phys. A 201, 357-368 (1973).

18. Nazarewicz, W., Riley, M. A. \& Garrett, J. D. Equilibrium Deformations and Excitation Energies of Single-Quasiproton Band Heads of Rare-Earth Nuclei. Nucl. Phys. A 512, 61-96 (1990).

19. Sakamoto, H. \& Kishimoto, T. Origin of the Multipole Pairing Interactions. Phys. Lett. B 245, 321-324 (1990).

20. Satuła, W. \& Wyss, R. Coherence of Nucleonic Motion in Superdeformed Nuclei: Towards an understanding of identical bands. Phys. Rev. C 50, 2888-2892 (1994)

21. Xu, F. R., Satuła, W. \& Wyss, R. Quadrupole Pairing Interaction and Signature Inversion. Nucl. Phys. A 669, 119-134 (2000).

22. Möller, P. \& Nix, J. R. Nuclear Pairing Models. Nucl. Phys. A 536, $20-60$ (1992). 23. Shimizu, Y. R. \& Matsuyanagi, K. Diabatic Mean-Field Description of Rotational Bands in Terms of the Selfconsistent Collective Coordinate Method. Prog. Theor. Phys. Supplement 141, 285-327 (2001) 
24. Strutinsky, V. M. Shell Effects in Nuclear Masses and Deformation Energies. Nucl. Phys. A 95, 420-442 (1967).

25. Myers, W. D. \& Swiatecki, W. J. Nuclear Masses and Deformations. Nucl. Phys. 81, 1-60 (1966).

26. Sahu, R. Collective Bands in ${ }^{84}$ Sr. Nucl. Phys. A 501, 311-318 (1989).

\section{Acknowledgments}

We would like to thank the operating staff of the HI-13 Tandem Accelerator at China Institute of Atomic Energy for providing the ${ }^{18} \mathrm{O}$ beam. The project is supported by

Suranaree University of Technology under contract No. 15/2553, the National Natura Science Foundation of China under Grant No. 11065001, and the Foundation of the

Education Department of Jiangxi Province under Grant No. GJJ12372.

\section{Author contributions}

S.S., G.H., S.W., X.K., Y.Y. and Y.W. wrote the main manuscript text, S.S. and G.H. prepared figures 1-2 and figure 4 and Z.B. prepared figure 3. X.W., L.Z., G.L. and C.H. designed experiment. All authors reviewed the manuscript.

\section{Additional information}

Competing financial interests: The authors declare no competing financial interests.

How to cite this article: Shen, S. et al. High-Spin States and Level Structure in Stable Nucleus Strontium-84. Sci. Rep. 3, 2740; DOI:10.1038/srep02740 (2013).

(c) (i) $(-)$ This work is licensed under a Creative Commons AttributionBY NC ND NonCommercial-NoDerivs 3.0 Unported license. To view a copy of this license, visit http://creativecommons.org/licenses/by-nc-nd/3.0 